NASA Technical Memorandum 101452

\title{
Free-Vibration Characteristics and Correlation of a Space Station Split-Blanket Solar Array
}

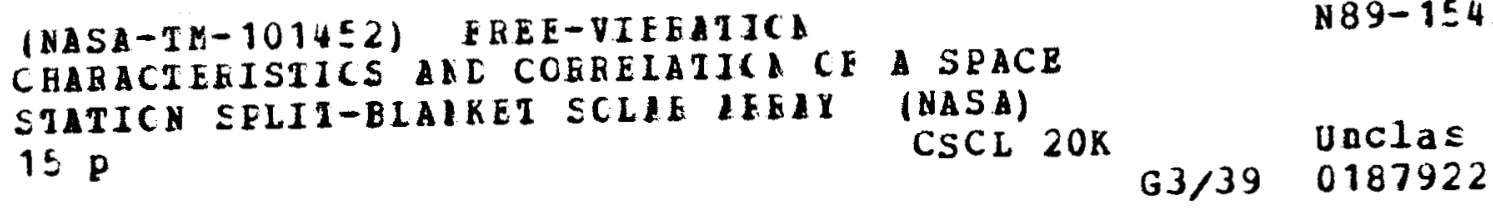

Kelly S. Carney and Francis J. Shaker

Lewis Research Center

Cleveland, Ohio

Prepared for the

30th Structures, Structural Dynamics and Materials Conference cosponsored by the AIAA, ASME, ASCE, AHS, and ACS

Mobile, Alabama, April 3-5, 1989

\section{N/SA}


Kelly S. Carney and Francis J. Shaker

National Aeronautics and Space Administration Lewis Research Center

Cleveland, Ohio 44135

\section{Abstract}

Two methods for studying the free-vibration characteristics of a large split-blanket solar array in a $0-g$ cantilevered configuration are presented. The $0-g$ configuration corresponds to an on-orbit configuration of the space station solar array. The first method applies the equations of continuum mechanics to determine the natural frequencies of the array; the second uses the finite element method program, MSC/NASTRAN. The stiffness matrix from the NASTRAN solution was found to be erroneously grounded. The results from the two methods are compared. It is concluded that the grounding does not seriously compromise the solution to the elastic modes of the solar array. However, the correct rigid body modes need to be included to obtain the correct dynamic model.

\section{Nomenclature}

b

EI

Ib mass polar moment of inertia per unit length

Itp mass moment of inertia of tip piece

$\bar{I}_{t p}$ tip piece inertia ratio (Eq. 19)

JG boom torsional stiffness

$\bar{k} \quad$ axial load parameter for bending (Eq. 15)

$\bar{k}_{t} \quad$ torsional stiffness factor (Eq. 19)

1 blanket and boom length

$\mathrm{Mb} \quad$ total mass of boom

$M_{m} \quad$ total blanket mass

$\bar{M}_{m} \quad$ mass ratio (Eq. 15)

$M_{t p} \quad$ mass of tip piece

$\bar{M}_{t p} \quad$ mass ratio (Eq. 15)

$M_{x}(x)$ torsional moment distribution along boom

$M_{y}(x)$ bending moment distribution along boom

$P \quad$ compressive preload in boom

$Q(x) \quad$ shear distribution along boom

$T \quad$ blanket tension per unit width

t time

$V(x, y, t)$ boom displacement

$W_{m} \quad$ total blanket weight

$\begin{array}{ll}W(x, y, t) & \text { blanket displacement } \\ x & \text { longitudinal coordinate } \\ y & \text { lateral coordinate } \\ \alpha_{1}, \alpha_{2}, \alpha_{3} & \text { characteristic values (Eq. 17) } \\ \bar{\beta} & \text { bending frequency parameter (Eq. 16) } \\ \bar{\beta}_{t} & \text { torsional frequency parameter (Eq. 20) } \\ \zeta & \text { transformed coordinate (Eq. 3) } \\ \mathrm{Pb}_{\mathrm{B}} & \text { mass per unit length of boom } \\ \rho_{\mathrm{m}} & \text { mass per unit length of blanket } \\ \omega & \text { circular frequency of vibration } \\ & \end{array}$

NASA's Space Station Freedom derives its electrical power from eight photovoltaic arrays. Each array is cantilevered off of the main space station truss as shown in Fig. 1. The eight arrays represent a significant amount of the mass and inertias of the space station outboard of the habitation and experimentation modules. The structural design of the photovoltaic arrays has been evolutionary. Several spacecraft and experiments have used similar designs in the past. A center extendable truss supports a pallet at the tip of the array. Two blanket substrates, with solar cells mounted on one side, are attached to this tip piece. The two blankets are arranged on either side of the extendable truss. The bottom of the blankets are constrained by negator springs which keep the blankets in constant tension. It is the constant tension which supplies the structural bending stiffness to the blankets. This configuration is illustrated in Fig. 2 .

Determining the dynamic response of the space station requires accurate models created using the finite element method. The position and flexibility of the photovoltaic arrays makes accurate prediction of their free-vibration characteristics particularly critical. This paper attempts to insure that the predicted frequencies are accurate by the following procedure. First, an exact solution of the equations of continuum mechanics for the natural frequencies of a split-blanket solar array is presented. The considered solar array contains several idealizing assumptions and is placed in a $0-g$ field. Second, $0-g$ natural frequencies of that same idealized split-blanket solar array are calculated using the finite element approach. There are several reasons why a check on this solution is desired. The tension supplied stiffness of the blanket must be modeled in the finite element method as a differential stiffness (geometric nonlinear) effect. Furthermore, as discussed in Ref. 1 , the resulting stiffness matrix is grounded in the rotational degrees of freedom. Therefore, in order to establish the 
acceptability of the finite element method for solving the $0-g$ split-blanket solar array problem, calculated cantilevered frequencies are compared to those calculated with the exact solution. In making this comparison the primary purpose of this paper is fulfilled. The first five natural frequencies are compared, which include three bending modes and two torsion modes.

\section{Solution of the Continuum Mechanics Equations for Normal Modes and Frequencies of the} Solar Array

\section{Previous Efforts}

The cantilevered modes and frequencies of a split-blanket solar array have been studied by several investigators. In Ref. 2 the cantilevered modes and frequencies of a split-blanket array in a 0 -g field were investigated by solving the differential equations governing the motion. This method results in transcendental equations that can be solved numerically for the frequencies. Reference 3 presents both a continuum mechanics approach and a Rayleigh-Ritz approach to calculating the natural modes and frequencies of split-blanket solar array in a $1-g$ field. The detailed derivation of the following equations are also contained in Ref. 3.

\section{Basic Assumptions}

For purposes of analysis a large split-blanket solar array is idealized as shown in Fig. 3. This figure shows the array consisting of three components: a center boom that supports the array (referred to as the extendable truss); a membrane substrate with solar cells attached to one side (referred to as the blanket); and a beam at the tip of the boom that transfers a tension load, $P$, from the boom to the substrate. The displacements of the boom and blanket, normal to the plane of the blanket, are denoted by $V(x, t)$ and $w(x, y, t)$, respectively. In developing the equations of motion for the array the following assumptions were made:

(1) The bending stiffness of the blanket, normal to its plane, is negligible so that the blanket behaves like a membrane in this direction.

(2) The tension distribution is uniform across the width of the blanket (i.e., the tip piece is rigid).

(3) Displacements are small, so that smalldisplacement theory is valid.

(4) Boom weight is negligible (in regards to the gravity gradient in a $1-g$ field), and the shear center coincides with the neutral axis of the boom. plane.

(5) The boom and the blanket lay in the same

Based on these assumptions the equations describing the motion of the array were developed.

\section{Equations of Motion}

The forces acting on an element of the blanket displaced an amount $W(x, y, t)$ from its static equilibrium configuration are shown in Fig. $4(a)$.
Applying Newton's second law of motion to this element yields the following equation:

$$
T \frac{\partial}{\partial x}\left(\frac{\partial W}{\partial x}\right)+\frac{\partial W}{\partial x} \frac{d T}{d x}=\frac{\rho_{m}}{b} \frac{\partial^{2} W}{\partial t^{2}}
$$

where $b$ is the blanket width and $\rho_{m}$ is the mass per unit length of blanket.

Now for a blanket hanging vertically, the tension at any point $x$ will be a superposition of the preload, $P$, transferred to the blanket from the boom and the weight of the blanket below the point $x$. That is,

$$
T(x)=\frac{P}{b}\left(1+\frac{W_{m}}{P}-\frac{\rho_{m} g}{P} x\right)
$$

where $W_{m}$ is the total blanket weight. In view of Eq. (2), Eq. (1) is transformed by making the following change of variables:

$$
\zeta^{2}=\frac{b}{p} T(x)=1+\frac{W_{m}}{p}-\frac{\rho_{m} g}{p} x
$$

From Eqs. (1) and (3) then

$$
\frac{\partial^{2} W}{\partial \zeta^{2}}+\frac{1}{\zeta} \frac{\partial W}{\partial \zeta}-\frac{4 \rho_{m} e^{2}}{P}\left(\frac{P}{W_{m}}\right)^{2} \frac{\partial^{2} W}{\partial t^{2}}=0
$$

Equation (4) represents the desired form for the equation of motion of the blanket.

The equation of motion in the transverse direction, for the beam element shown in Fig. $4(\mathrm{~b})$, as given by beam theory (Ref. 4 ), is

$$
Q(x)=-E I\left(\frac{\partial^{3} V}{\partial x^{3}}+\frac{P}{E I} \frac{\partial V}{\partial x}\right)
$$

In this manner it can be shown (Ref. 5) that the equation governing the bending motion of the boom is given by

$$
\frac{\partial^{4} V}{\partial x^{4}}+\frac{P}{E \bar{I}} \frac{\partial^{2} V}{\partial x^{2}}+\frac{\rho_{b}}{E I} \frac{\partial^{2} V}{\partial t^{2}}=0
$$

In addition to the bending motion described by Eq. (6), the boom can also experience a rotational motion about its centerline. The equation governing this motion is developed in numerous texts on vibration theory (e.g., Ref. 6) and is given by

$$
\frac{\partial^{2} \theta}{\partial x^{2}}-\frac{I_{b}}{J G} \frac{\partial^{2} \theta}{\partial t^{2}}=0
$$

where $\theta$ is the rotational angle of the boom cross section, $I_{b}$ is the mass polar moment of inertia per unit length, and $J G$ is the torsional stiffness of the boom. Equations (5) to (7) represent the required relations for the boom.

The final set of equations are the equations of motion for the tip piece. The forces acting on the tip piece are shown in Fig. 5. Applying Newton's second law of motion for torces in the 
$z$-direction and moments about an axis parallel to the $x$-axis and passing through the center of gravity of the tip piece yields the following two equations:

$$
\begin{array}{r}
Q(l, t)+\int_{-b / 2}^{b / 2} \frac{P}{b} \frac{\partial W(l, y, t)}{\partial x} d y+M_{t p} \frac{\partial^{2} V(l, t)}{\partial t^{2}} \\
=0 \\
M_{x}(l, t)+\int_{-b / 2}^{b / 2} \frac{P}{b} \frac{\partial W(l, y, t)}{\partial x} d y+I_{t p} \frac{d^{2} \alpha}{d t^{2}}=0
\end{array}
$$

where $M_{t p}$ is the mass of the tip piece and Itp is the mass moment of inertia about its center of gravity. Equations (10) and (11) can be written in terms of displacement variables. This results in the following form for the tip piece equations.

$$
\begin{aligned}
& \frac{\partial^{3} V(l, t)}{\partial x^{3}}+\frac{P}{E I} \frac{\partial V(l, t)}{\partial x}+\frac{1}{2 l b}\left(\frac{P}{E I}\right)\left(\frac{W}{P}\right) \\
& \quad x \int_{-b / 2}^{b / 2} \frac{\partial W(1, y, t)}{\partial \zeta} d y-\frac{M}{E I} \frac{\partial^{2} V(l, t)}{\partial t^{2}}=0
\end{aligned}
$$

$$
\begin{array}{r}
\frac{\partial \theta(l, t)}{\partial x}-\frac{1}{2 \ell b}\left(\frac{P}{J G}\right)\left(\frac{W}{P}\right) \int_{-b / 2}^{b / 2} y \frac{\partial W(1, y, t)}{\partial \zeta} d y \\
+\frac{1 t p}{J G} \frac{d^{2} \alpha}{d t^{2}}=0
\end{array}
$$

Equations (10) and (11) represent the final form of the equations of motion of the tip piece. These equations together with Eqs. (4), (6), and (7) represent the motion equations for the complete solar array. The displacement variables in these equations must satisfy certain boundary and compatibility relations. These conditions are given next.

\section{Boundary Conditions and Compatibility Relations}

At the fixed end of the array the displacements and rotations of the array elements are all zero. At this end, $x=0$ and it follows from Eq. (3) that $\zeta_{0}=\sqrt{1+\left(W_{m} / P\right)}$. Thus, the boundary conditions at the fixed end will be as follows:

$$
\left.\begin{array}{c}
V(0, t)=0 \\
\frac{\partial V}{\partial x}(0, t)=0 \\
\theta(0, t)=0 \\
W\left(\zeta_{0}, y, t\right)=0
\end{array}\right\}
$$

At the free end of the array the displacements and rotations of the components must be compatible. At this end $x=1$; and from Eq. (3), $\zeta=1$. In add $i-$ tion, the moment at the tip of the boom is zero. Thus, the boundary and compatibility relations at the free end are as follows:

$$
\begin{gathered}
\theta(l, t)=\alpha \\
W(1,0, t)=V(l, t) \\
W(1, y, t)=W(1,0, t)+y \alpha \\
\frac{\partial^{2} V}{\partial x^{2}}(l, t)=0
\end{gathered}
$$

Equations (10) to (13) represent the complete set of relations that must be satisfied by the solutions to Eqs. (4), (6), and (7).

\section{Exact Solution to the Equations of Motion}

The derivation of the characteristic equations for the bending and torsional frequencies is described in detail in Ref. 3. Included in this reference are the solutions for a solar array in a $1-g$ ground test configuration. For the case of a solar array in a $0-g$ field (i.e., an on-orbit configuration), these characteristic equations are determined by taking the limit of the functions in the $1-g$ equations as the blanket weight $W_{m}$ approaches zero. This process is also described in detail in Ref. 3. The characteristic equation for the bending frequencies of the solar array in a $0-g$ configuration can be shown to be:

$$
\begin{aligned}
\left(\bar{M}_{t p} \bar{\beta}^{4} \sin \alpha_{3}-\bar{k}^{2} \alpha_{3} \cos \alpha_{3}\right)\left[\left(\alpha_{1}^{2}+\alpha_{2}^{2}\right)\right. \\
\left.\quad \times\left(\alpha_{2} \sinh \alpha_{1} \cos \alpha_{2}-\alpha_{1} \cosh \alpha_{1} \sin \alpha_{2}\right)\right] \\
\quad+\sin \alpha_{3}\left[2 \bar{\beta}^{6}+\bar{\beta}^{2}\left(2 \bar{\beta}^{4}+\bar{k}^{4}\right)\right. \\
\left.\quad \times \cosh \alpha_{1} \cos \alpha_{2}-\bar{\beta}^{4} \bar{k}^{2} \sinh \alpha_{1} \sin \alpha_{2}\right] \\
\quad=0
\end{aligned}
$$

The parameters used in Eq. (14) have been nondimensionalized by the use of the following relationships:

$$
\bar{M}_{t p}=\frac{M_{t p}}{M_{b}}, \quad \bar{M}_{m}=\frac{M_{m}}{M_{b}}, \quad \bar{k}^{2}=\frac{P \ell^{2}}{E I}
$$

with the bending frequency parameter defined to be

$$
\bar{\beta}^{4}=\frac{M_{b} \ell^{3}}{E I} \omega^{2}
$$

and the $\alpha$ functions defined as

$$
\left.\begin{array}{c}
\alpha_{1}=\left(-\frac{\bar{k}^{2}}{2}+\sqrt{\frac{\bar{k}^{4}}{4}+\bar{\beta}^{4}}\right)^{1 / 2} \\
\alpha_{2}=\left(-\frac{\bar{k}^{2}}{2}+\sqrt{\frac{\bar{k}^{4}}{4}+\bar{\beta}^{4}}\right)^{1 / 2} \\
\alpha_{3}=\frac{\bar{M}_{m}^{1 / 2}}{\bar{k}} \bar{\beta}^{2}
\end{array}\right\}
$$


The characteristic equation for the torsional frequencies of the solar array can be shown to be:

$$
\cos \bar{\beta}_{t}+12\left(\frac{1}{\bar{k}_{t}^{2} \bar{\beta}_{t}}-\bar{I}_{t p} \bar{\beta}_{t}\right) \sin \bar{\beta}_{t}=0
$$

The parameters used in Eq. (18) have been nondimensionalized by these following relationships:

$$
\bar{k}_{t}^{2}=\frac{P b^{2}}{J G}, \quad \bar{I}_{t p}=\frac{I_{t p}}{M_{m} b^{2}}
$$

with the torsional frequency parameter defined as

$$
\bar{\beta}_{t}^{2}=\frac{M_{m} l}{P} \omega^{2}
$$

It can be shown that Eqs. (14) and (18) are analogous to the frequency equations in Ref. 7 , which were developed in a straightforward manner for the $0-g$ configuration.

\section{Summary of Continuum Mechanics Approach}

In the previous sections the equations governing the frequencies of a split-blanket solar array, which were determined by solving the differential equations governing the motion of the system, were presented. The results showed that the array will exhibit symmetric or bending modes and antisymmetric or torsional modes of vibration (Fig. 6). The frequencies of the bending modes can be found by solving $\mathrm{Eq}$. (14) for an array in a $0-g$ field. Similarly, the torsional mode frequencies can be determined from Eq. (18). In both of these cases a highly transcendental equation involving trigonometric and hyperbolic functions must be solved numerically to obtain a solution. The frequencies of the array in a $0-g$ field with parameters matching those for the space station solar array were calculated and will be presented in the comparison section.

\section{Finite Element Approach}

\section{Finite Element Modeling}

A configuration of the space station solar array was modeled using the finite element program, MSC/NASTRAN. There are several differences between the space station solar array model and our idealized solar array model because of the assumptions discussed in the previous section. The idealized solar array finite element model has no local flexibility or masses. In the actual space station array model there are local flexibilities which represent joints and masses which represent electronic packages. The idealized solar array was modeled to match the structure solved for in the exact solution as closely as possible.

The substrate (blanket) of split-blanket solar arrays has no inherent bending stiffness. The bending stiffness is derived from being stretched by a constant tension. This effect is analogous to the stiffness achieved when a drum head is stretched. The blanket of the space station idealized solar array is tensioned by applying forces directly to the end of this blanket. The direction of the forces is maintained in the plane of the blanket. There is no supporting structure modeled at the bottom of the blanket where the forces are applied. In the actual design of the space station solar array there is a blanket box that supports the bottom of the blanket and the tensioning mechanisms. The blanket itself is represented as differential stiffness membrane elements. The membrane elements used in this analysis are the standard MSC/NASTRAN CQUAD4 elements which have their bending stiffness defined as zero. The properties for these elements were derived from an EAL finite element model which was supplied by Lockheed Missiles and Space Company. They are consistent with the proposal Lockheed made to the space station project for the solar arrays (Ref. 8).

The membrane elements which define the blanket are connected to a top structural member. In order to be consistent with the assumptions that were made in defining the exact solution, this tip piece was modeled using rigid elements and was connected rigidly to the center boom. The center boom was modeled using standard (CBEAM) beam elements. Normally the center beam is cantilevered at the base, but this constraint can be removed to calculate the rigid body modes. This finite element model is illustrated in Fig. 7. The various structural parameters that were used to define this model are given in Table 1. These same parameters were used to calculate frequencies in the exact solution. Due to programmatic changes, there are small differences in size between these parameters and the current space station solar array specifications.

\section{Finite Element Solution}

For the static tensioning of the model to supply the bending stiffness to the blanket, differential stiffness capabilities must be utilized. The differential stiffness effects in the case of the solar array blanket are analogous to the stiffness achieved when a drum head is stretched. In a finite element solution the differential stiffness terms are added to the element stiffness matrix. In MSC/NASTRAN this can be accomplished in the standard solution sequences. The static tensioning of the blanket takes place in MSC/NASTRAN's geometric nonlinear solution 64 (Ref. 9). The tension applied to each blanket in the actual space station solar array is $75 \mathrm{lb}$ (total tension being 150 lb). For the purposes of this study the load is varied from $1001 \mathrm{~b}$ to the buckling lad of the combined system.

The geometric nonlinear solution calculates an initial approximation for the differential stiffness effects that result from the static tensioning. These effects are added to standard element stiffness matrix. Through the use of additional subcases the solution to the geometric nonlinear static problem is iterated upon until the applied load and the internal loading is balanced. At every iteration the geometry, the external loading and consequently the internal loading and the stiffness matrix is updated. After the final iteration, when the solution has successfully converged, the stiffness matrix is retained. This stiffness matrix is utilized by the normal modes solution 63 sequence. The system natural frequencies and mode shapes, using the updated stiffness matrix which include differential stiffness effects, are calculated in the standard normal modes solution. 


\section{Grounding}

The normal modes and frequencies, which include differential stiffness effects, can be calculated for both the cantilevered configuration and the free-free configuration. When the normal modes and frequencies were calculated in a free-free situation, only three rigid body modes were found to exist. These were the three translational rigid body modes. The three rotational rigid body modes do not exist for this differential stiffness finite element model. Rigid body translations and rotations were also applied to the stiffness matrix of the model. The rigid body rotations generated large internal forces and moments. Therefore, the stiffness matrix that results from the geometric nonlinear solution was determined to be grounded.

An effort was made to determine the cause of this problem. The finite element differential stiffness matrix that was created by MSC/NASTRAN was found to be identical to the stiffness matrix formulated by Martin (Ref. 10). In Ref. 1 it was pointed out that Martin's formulation of the differential stiffness matrix would not have the rotational rigid body modes. However, the grounding of the entire model that occurs as a result of this phenomena was not identified. Therefore, it was concluded that the differential stiffness terms which are added to the element stiffness matrix when the model is statically tensioned grounds the stiffness matrix. This effort is documented in Ref. 11

Generating and implementing a new element differential stiffness matrix to include the rigid body rotations would not be feasible as a short term solution. Reformulating the differential stiffness membrane finite element would be time consuming and perhaps unneeded, if the missing rotational rigid body modes are relatively easy to calculate. If the cantilevered elastic modes are calculated, the correct rigid body modes can be appended to the matrix containing the elastic modes. The resulting set of matrices is then equivalent to a standard Craig-Bampton component mode representation of the solar array. However, this model will be correct only if the grounding has not affected the elastic modes. To this end the results from the finite element solution were compared to the exact solution.

\section{Comparison Between Exact and Finite Element Solutions}

The lack of rotational rigid body modes in the finite element solution does not prove that the predicted elastic frequencies are in error. To determine the finite element method's accuracy its frequencies were compared to those calculated using the exact solution. Two separate FORTRAN programs were written to calculate the bending and torsional frequencies predicted by the exact solution. The equations used to calculate these frequencies were Eqs. (14) and (18), respectively. The magnitude of the tension in the solar array for both the NASTRAN and the exact solution was varied up to the first buckling load of the array. The first buckling load of this idealized space station solar array is $21301 \mathrm{~b}$. The operating point of the actual space station solar array, as mentioned previously, is $150 \mathrm{lb}$, or 7 percent of the critical buckling load.
The calculated frequencies from the two different solutions were compared for the first three bending modes and the first two torsion modes. A graph of the tension load versus frequency of the bending modes is shown in Fig. 8. As the tension load increases, the frequencies of the solar array begin to rise due to the increased stiffness in the blanket. As the tension continues to increase, the compression in the center mast begins to cause the frequencies to drop. The fundamental bending frequency goes to zero at the first buckling mode of the beam. Figure 9 is a graph of the percent difference between the exact solution and the finite element solution of the first bending mode. The reason for the increased difference between the solutions as the load approaches the buckling load has not been determined at this time. Figure 10 plots the frequencies of the first two torsion modes against the tension load. Figure 11 is the percent difference between the exact and finite element solutions of the first torsion mode. The tension load was normalized to the first buckling load of the system in all the figures.

The agreement between the finite element solution and the exact solution is excellent in the range of interest. Several other finite element mesh densities were al so used to calculate frequencies. It was found that the accuracy of this problem was not sensitive to mesh density. The overall good correlation is not necessarily a general result. Several cases were created where the grounding significantly affected the elastic modes predicted by the finite element solution. As a general rule, a procedure such as was followed herein, comparing an exact solution to finite element solutions, should be utilized when using differential stiffness.

\section{Conclusions}

In the loading range of interest, there is excellent agreement between the finite element solution and the exact solution. Therefore, the elastic modes of the finite element solution are accurate. The correct rigid body modes can be added to the finite element calculated elastic modes to form a Craig-Bampton component mode representation which can then be used in subsequent analyses. The generally good agreement between the two solutions should not be construed to establish that grounding caused by differential effects can never be a problem. There could be situations where the grounding alters the elastic modes significantly. Whether this is the case in any specific problem would have to be determined on an individual basis.

\section{References}

1. Collar, A.R. and Simpson, A.: Matrices and Engineering Dynamics, Halsted Press, New York, 1987.

2. Hughes, P.C. and Garg, S.C.: Dynamics of Large Flexible Solar Arrays and Application to Spacecraft Attitude Control System Design." UTIAS-179, Toronto University, 1973.

3. Shaker, F.J.: "Free-Vibration Characteristics of a Large Split-Blanket Solar Array in a 1-g Field," NASA TN D-8376, 1976. 
4. Timoshenko, S.P. and Gere, J.M.: Theory of Elastic Stability, 2nd edition, McGraw-Hill Book Co., New York, 1961.

5. Shaker, F.J.: "Effect of Axial Load on Mode Shapes and Frequencies of Beams," NASA TN D-8109, 1975.

6. Meirovitch, L.: Analytical Methods in Vibration. MacMillan Co., New York, 1967.

7. Harrison, T.D.: "Communications Technology Satellite Deployed Solar Array Dynamics Tests," CRC-1264, Communications Research Centre, Ottawa, Canada, 1975

8. "Proposal for Space Station Program WP-04, Solar Array Assemblies, Option One, Vol. 1," LMSC-F177627, Lockheed Missiles and Space Co. Sunnyvale, CA, 1987.
9. Joseph, J.A., ed.: MSC/NASTRAN Application Manual, The MacNeal-Schwendler Corp., 1984.

10. Martin, H.C.: "On the Derivation of Stiffness Matrices for the Analysis of Large Deflection and Stability Problems," Matrix Methods in Structural Mechanics, AFFDL-TR-66-80, R.M. Bader, et al., eds., 1966, pp. 697-716. (Avail. NTIS, AD-646300.)

11. Bosela, P.: "Limitations of Current Nonlinear Finite Element Methods in Dynamic Analys is of Solar Arrays," MSC Users' Conference, Los Angeles, CA, Mar. 1989.

TABLE 1. - PARAMETERS WHICH DEFINE THE IDEALIZED SOLAR ARRAY STRUCTURE

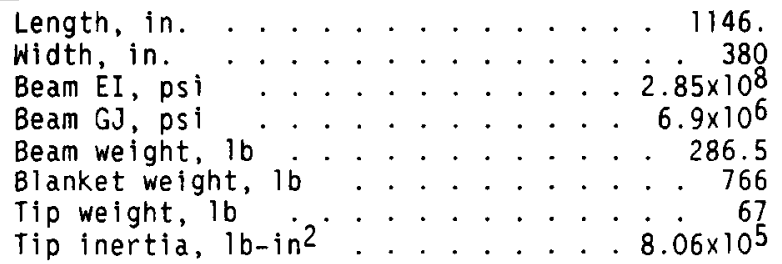




\section{ORIGINAL PAGE IS \\ OF POOR QUALITY}

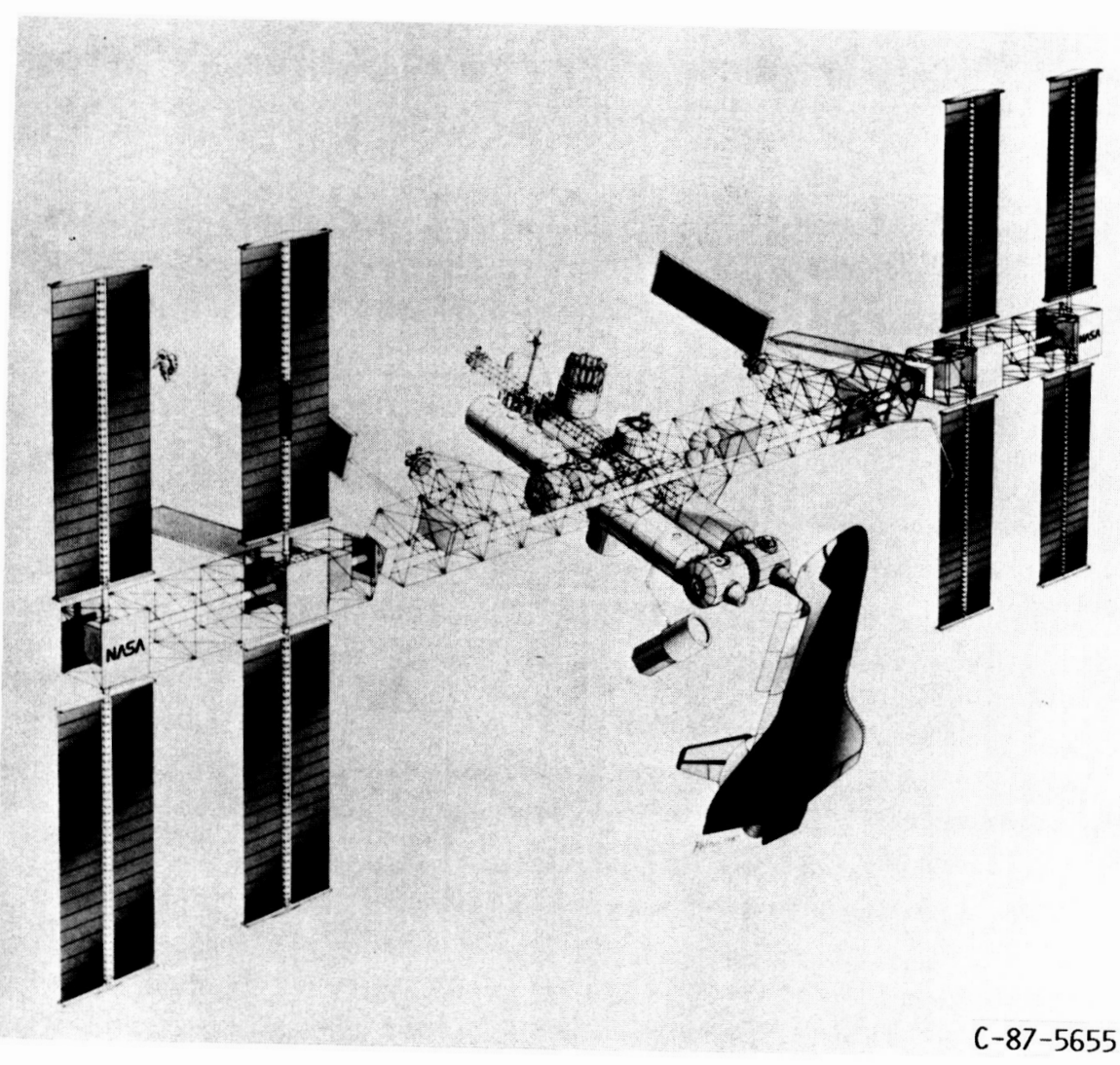

FIGURE 1. - SPACE STATION FREEDOM. 


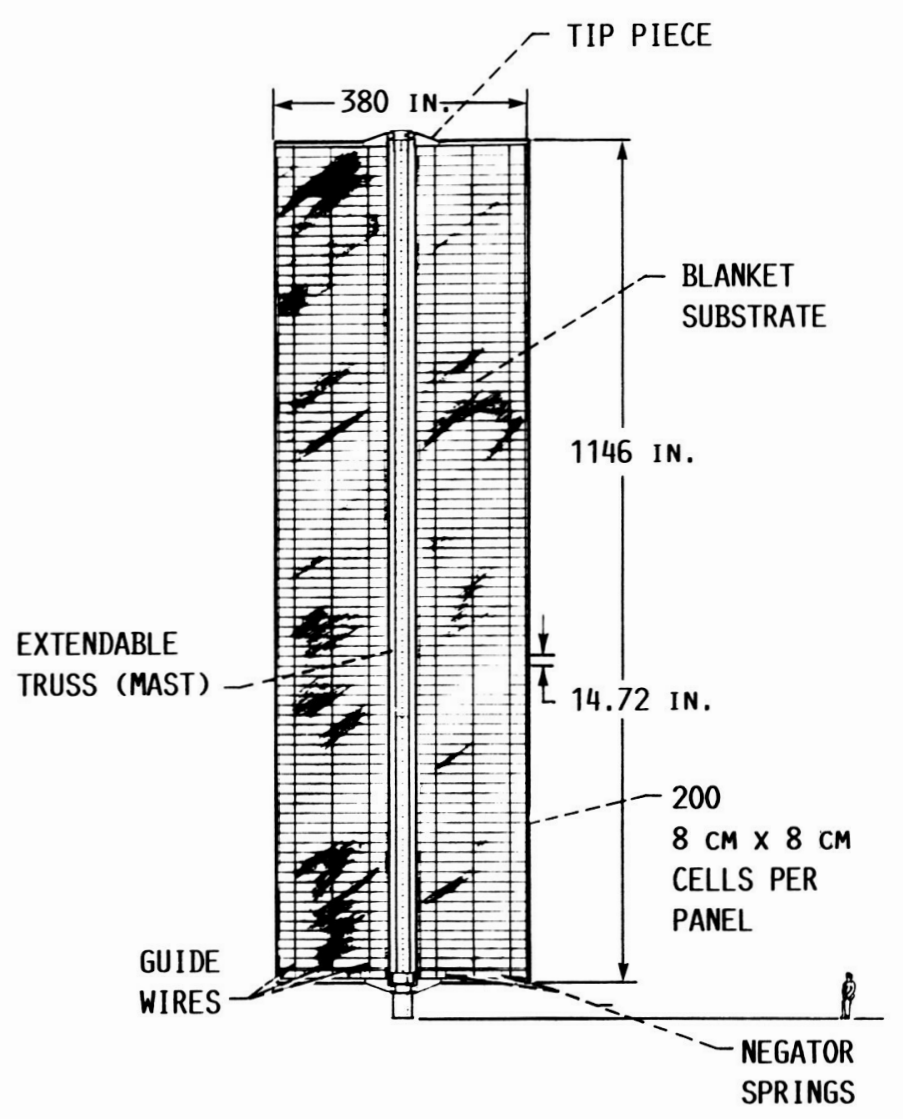

FIGURE 2. - SPACE STATION SPLIT BLANKET SOLAR ARRAY.

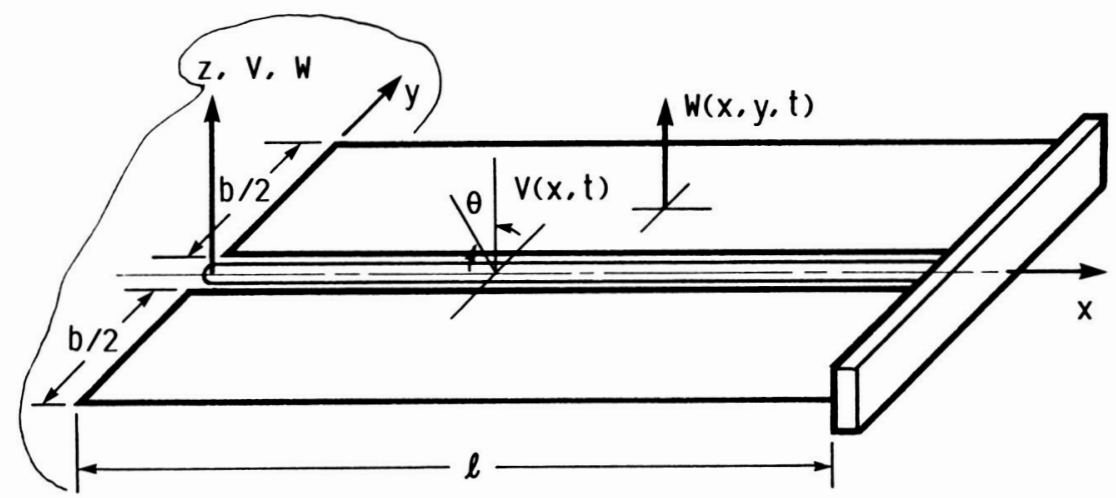

FIGURE 3. - GEOMETRY AND COORDINATE SYSTEM FOR A LARGE SOLAR ARRAY WITH A SPLIT BLANKET. 


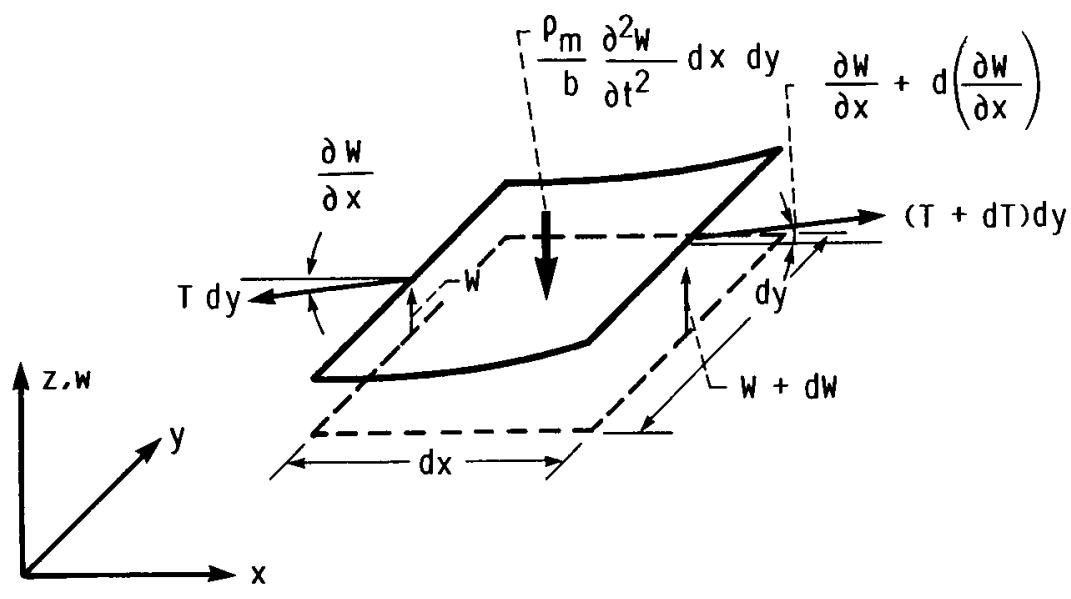

(a) BLANKET ELEMENT SHOWING FORCES AND DISPLACEMENTS.

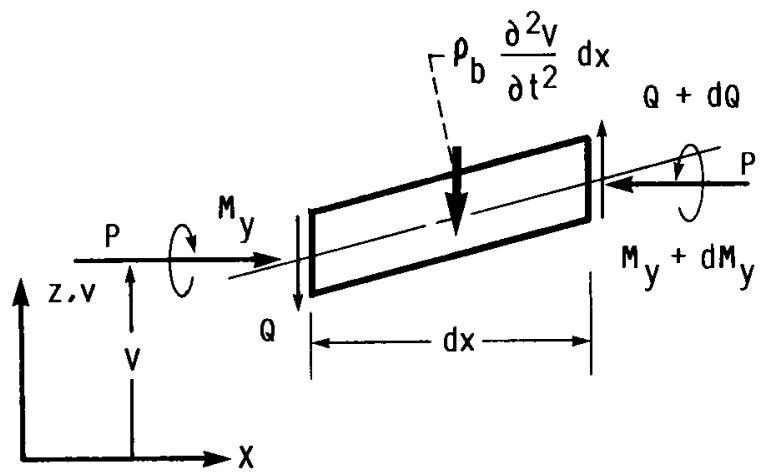

(b) BEAM ELEMENT SHOWING FORCES, MOMENTS, AND DISPLACEMENTS.

FIGURE 4. - FORCES ACTING ON BLANKET AND BEAM. 


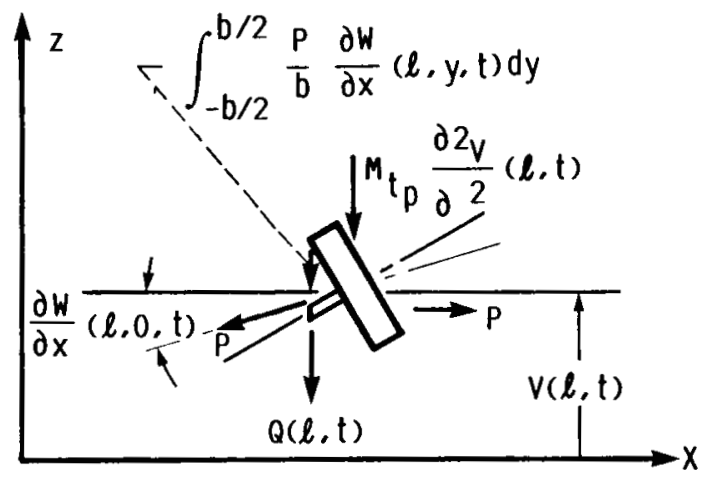

(a) FORCES AND DISPLACEMENT OF TIP PIECE IN $X-Z$ PLANE.

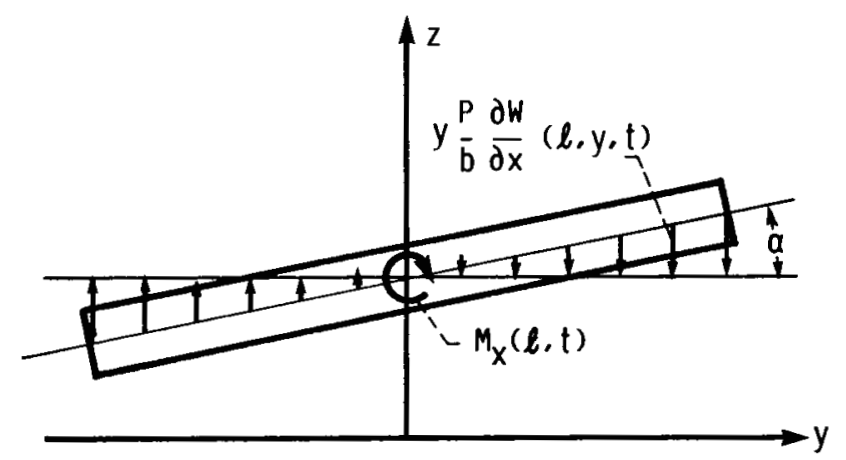

(a) FORCES AND DISPLACEMENTS OF TIP PIECE IN $y-z$ PLANE. (FOR CLARITY THE UNIFORM LOAD (P/b) $\partial W / \partial x(\boldsymbol{l}, \mathrm{t})$ IS NOT SHOWN, )

FIGURE 5. - FORCES AND DISPLACEMENTS OF TIP PIECE. 


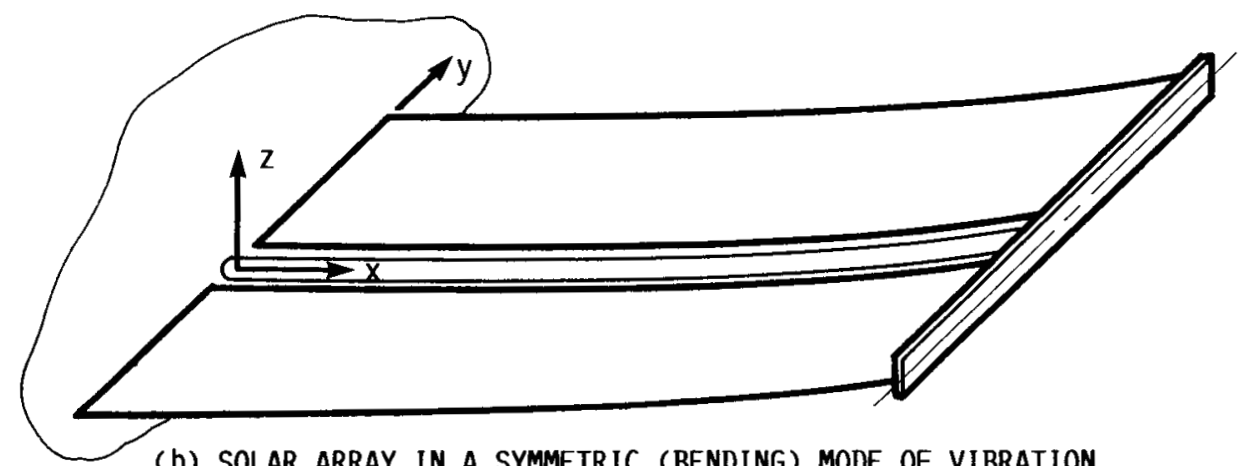

(b) SOLAR ARRAY IN A SYMMETRIC (BENDING) MODE OF VIBRATION.

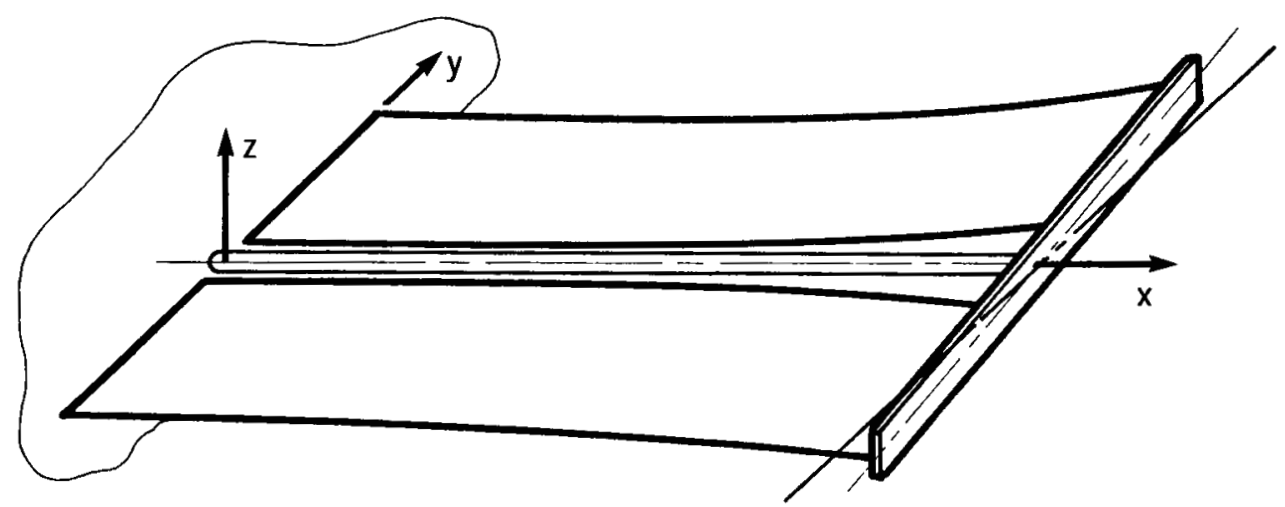

(b) SOLAR ARRAY IN A ANTISYMMETRIC (TORSIONAL) MODE OF VIBRATION.

FIGURE 6. - BENDING AND TORSIONAL MODES OF VIBRATION. 


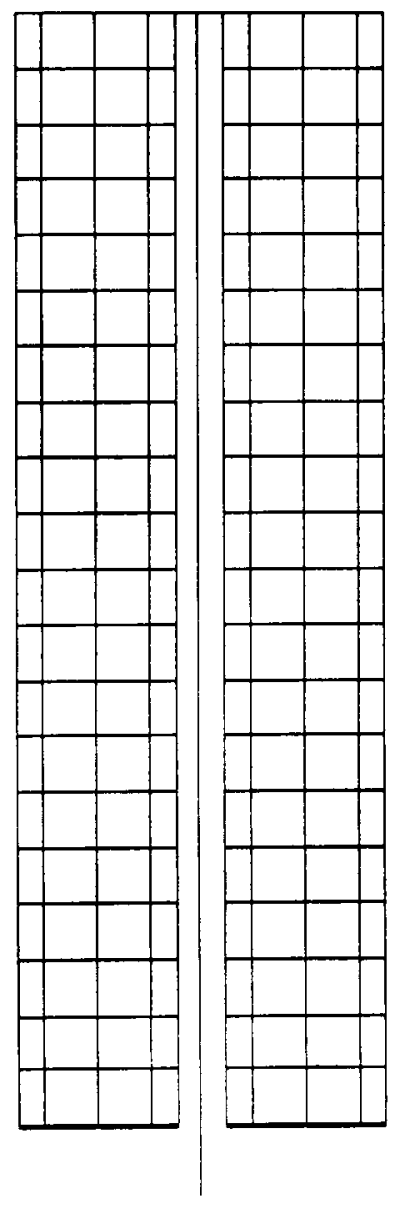

FIGURE 7. - FINITE ELEMENT MODEL OF IDEALIZED SPACE STATION SOLAR ARRAY.

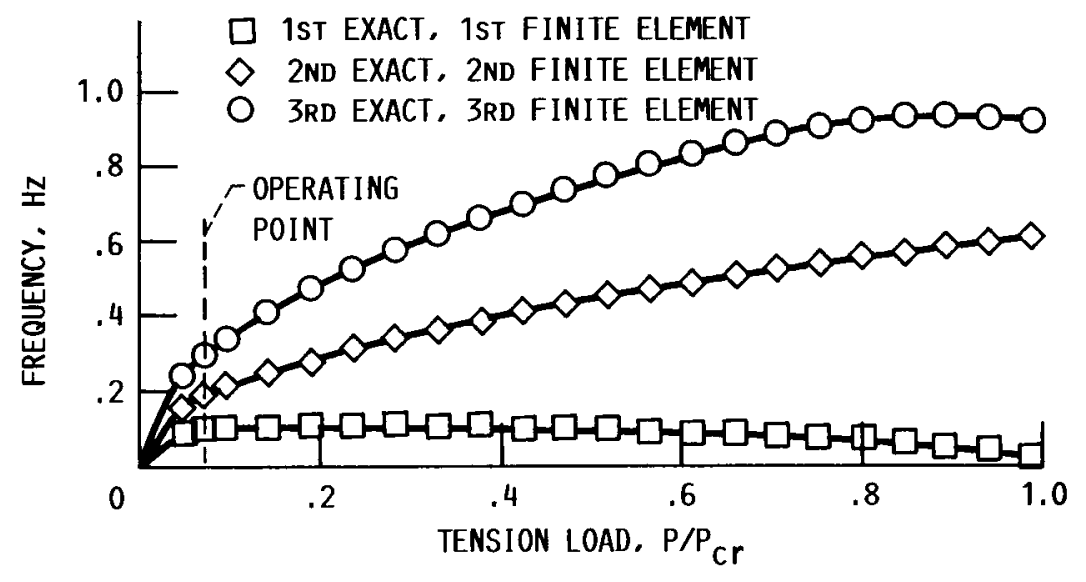

FIGURE 8. - BENDING FREQUENCIES VERSUS LOAD, EXACT AND FINITE ELEMENT SOLUTION OF AN IDEALIZED PHOTOVOLTAIC ARRAY. 


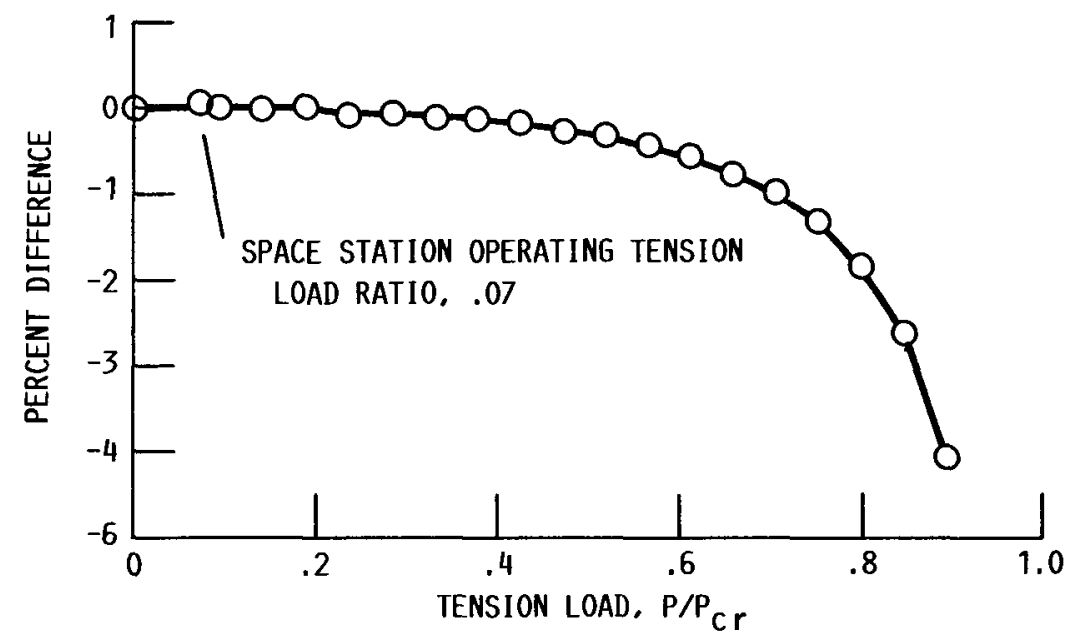

FIGURE 9. - PERCENT DIFFERENCE BETWEEN EXACT AND FINITE ELEMENT SOLUTION OF 1ST BENDING MODE VERSUS TENSION LOAD.

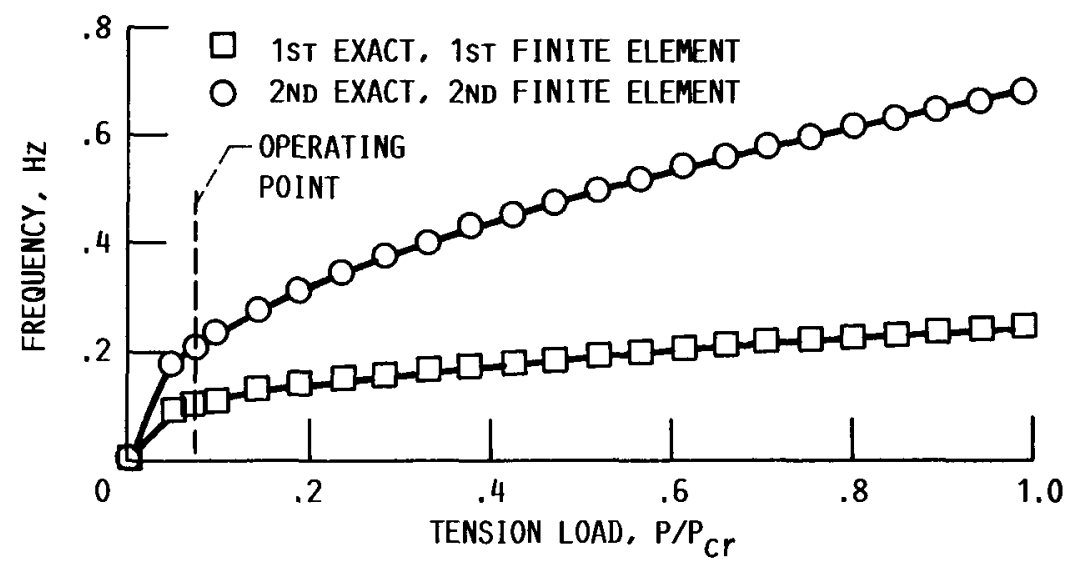

FIGURE 10. - TORSION FREQUENCIES VERSUS LOAD, EXACT AND FINITE ELEMENT SOLUTION.

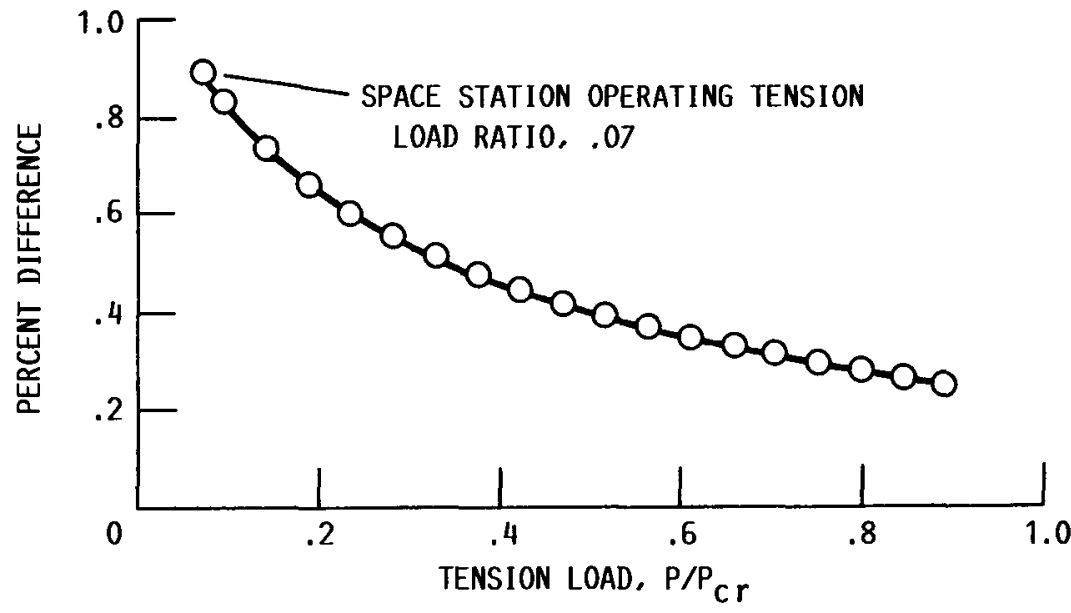

FIGURE 11. - PERCENT DIFFERENCE BETWEEN EXACT AND FINITE ELEMENT SOLUTION OF 1ST TORSION MODE VERSUS TENSION LOAD. 


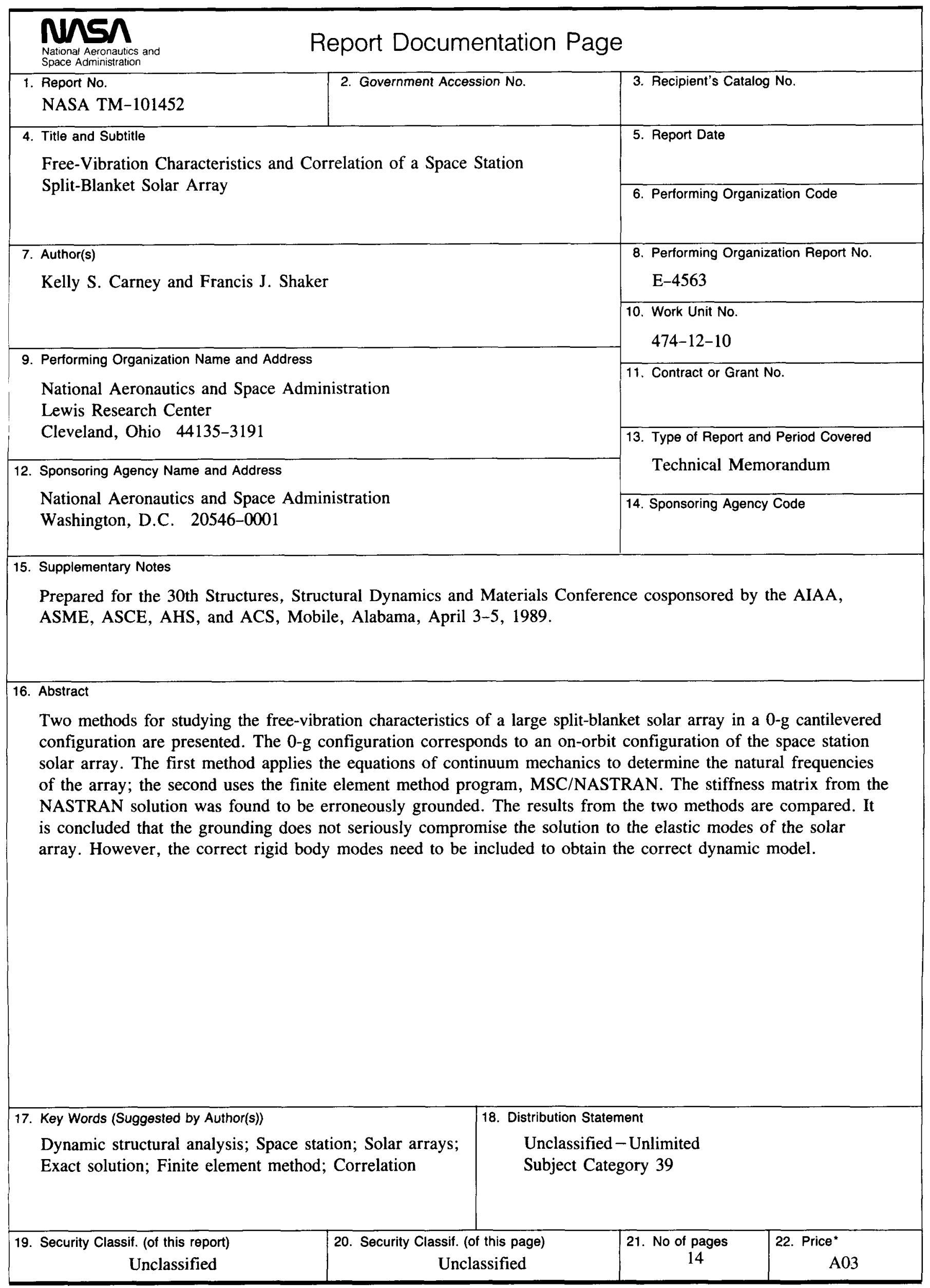

